\title{
Ubicación de sitios arqueológicos a través de nuevas tecnologías de la información geográfica, valle de Otoro
}

Vito Veliz, César Rodríguez y Cristina Argueta ${ }^{1}$

\section{RESUMEN}

Esta primera fase del proyecto Ubicación de sitios arqueológicos a través de nuevas tecnologías de la información geográfica, tenía por objetivo ubicar sitios arqueológicos en el valle de Otoro, Intibucá, y determinar posibles alineamientos astronómicos con respecto a la posición del Sol en determinadas épocas del año. Se ha procedido a registrar toda la información bibliográfica y por medio de visitas de campo se ha comprobado el estado de los sitios y su localización geográfica, actualizando la información arqueológica disponible.

Aunque para Otoro se disponía de un conteo de sitios arqueológicos (12 sitios), se deseaba comprobar su ubicación, guiándose con los mapas topográficos y las lecturas con GPS que se hicieron al momento de la visita. En esta se registraron 13 sitios arqueológicos que se encuentran en el municipio de Jesús de Otoro, valle de Otoro, para lo cual se utilizó un GPS Mobile Mapper 6, marca Magellan, con un margen de error de 1 metro. Se registró fotográficamente cada sitio, se analizó su estado, características y estructuras visibles. También se generó una base de datos y cartografía temática sobre la ubicación de estos sitios en el valle de Otoro, proceso que es importante para la siguiente fase del proyecto.

El proyecto permitió identificar y registrar un nuevo e importante sitio arqueológico en la zona de estudio llamado Guayamán, el cual está en proceso de deterioro. Una vez lograda la ubicación de los yacimientos arqueológicos, se elaboró cartografía temática, se buscó el significado arqueoastronómico de cada sitio; es decir, se analizó la relación de cada sitio con algún astro, en especial con el Sol, para determinar posibles alineamientos astronómicos con respecto a la posición del Sol en determinadas épocas del año.

Palabras clave: ubicación, sitios arqueológicos, sistemas de información geográfica, teledetección, arqueoastronomía.

Universidad Nacional Autónoma de Honduras. Facultad de Ciencias Espaciales, FACES-UNAH-DAQAC. Correo electrónico: vitoveliz@yahoo.com; cesaronca@gmail.com; cristina.argueta@unah.edu.hn 


\section{ABSTRACT}

This first phase of the project Location of archaeological sites across new technologies of the geographical information, had for aim locate archaeological sites in the Valley of Otoro, Intibucá and determine possible astronomic alignments with regard to the position of the Sun in certain epochs of the year. One has proceeded to register all the bibliographical information and by means of field visits there has been verified the condition of the sites and his geographical location, updating the archaeological available information.

Though for Otoro it was the arranging of a count of archaeological sites (12 sites), one wanted to verify his location, guided with the topographic maps and the readings with GPS that were done to the moment of the visit. In this one there were registered 13 archaeological sites that they found in Jesus de Otoro's municipality, Otoro's Valley, using a GPS Mobile Mapper 6, it marks Magellan, with a margin of mistake of 1 meter. Every site was registered photographically, there were analyzed its condition, characteristics and visible structures. Also there was generated a base of information and thematic cartography on the location of these sites in Otoro's Valley, process that is important for the following phase of the project.

The project allowed to identify and to register a new and important archaeological site in the zone of study called Guayamán, which is in process of deterioration. Once achieved the location of the archaeological deposits, I elaborated thematic cartography, The archeoastronomic meaning was looked of every site; it is to say, there was analyzed the relation of every site by some star, especially by the Sun, to determine possible astronomic alignments with regard to the position of the Sun in certain epochs of the year.

Keywords: Location, archaeological sites, geographic information systems, remote sensing, archaeoastronomy.

\section{INTRODUCCIÓN}

La aplicación de sistemas de información geográfica y técnicas de teledetección para el reconocimiento de restos arqueológicos arquitectónicos en la superficie terrestre es algo que al inicio de esta investigación no se había utilizado en Honduras. Tanto en los Estados Unidos (Lyons y Mathien, 1980) como en Europa (Campana, Stefano y Forte, 2006), los proyectos utilizando esta técnica han dado resultados muy positivos, pero las circunstancias y la naturaleza de los restos arqueológicos no son siempre comparables. En consecuencia, era necesario hacer 
pruebas y encontrar las técnicas apropiadas para poder reconocer esos restos arqueológicos en Honduras, utilizando SIG y la tecnología avanzada de teledetección.

Alrededor del mundo se ha encontrado evidencia de la influencia de los astros sobre el ser humano y sobre algunas de sus obras. Muchos investigadores se han dado a la tarea de estudiar y descubrir las evidencias que los antiguos dejaron sobre sus conocimientos astronómicos y sobre la influencia de los cuerpos celestes en la mentalidad y en ciertas obras humanas (Aveni, 1981; Pineda de Carías, Véliz y Agurcia Fasquelle, 2002).

En vista de la presencia de distintos nichos ecológicos en Honduras, desde el inicio de este proyecto se decidió considerar a estos; lo cual es un aspecto muy necesario a tomar en cuenta porque, dependiendo de eso, la contextura o colores de las imágenes satelitales estarán sujetos a esa circunstancia. Asimismo, la cobertura del terreno determinará la naturaleza de los pixeles que compondrán la imagen. Siendo un valle, Jesús de Otoro presenta un aspecto semiárido, una cobertura vegetal variada y ya se sabe que ahí existen varios sitios arqueológicos. Todo esto le da el carácter de una zona de ensayo, de prueba, cuyos resultados servirán para aplicarlos en zonas similares en el resto del país (Véliz, 1983).

Investigaciones previas marcaron el desarrollo de este estudio: Juan Gregorio Rejas Ayuga y Vito Veliz presentaron en 2008 el proyecto denominado: Desarrollo en nuevas tecnologías para la protección y preservación del patrimonio cultural y hábitat humanos de Honduras. Como continuación al desarrollo de esta investigación, se presentaron los avances del Proyecto teledetección, en el cual se recopilo información de los sitios arqueológicos de Honduras, Veliz (2009). Posteriormente, Véliz (2010) presenta el proyecto de investigación Arqueología de las zonas de estudio de un proyecto de teledetección en la República de Honduras.

Uno de los procesos utilizados generalmente para asegurar el aprovechamiento de las técnicas espaciales de reconocimiento es conocer los sitios arqueológicos en tierra, ubicarlos en mapas topográficos y en la imagen satelital y analizar los rasgos del punto representativo de esos restos en la imagen satelital. Después, se describen los rasgos representativos de esos sitios arqueológicos en la imagen satelital, para poder reconocer esos mismos rasgos en otros puntos o zonas de las imágenes satelitales.

En esta primera fase del proyecto general, se está dando el primer paso en ese proceso, que es la obtención de información general sobre la ubicación de sitios 
arqueológicos en el valle de Otoro, por medio de una revisión de la literatura arqueológica de esa zona. De igual modo, se aprovechó el verano para verificar las lecturas de ubicación con GPS.

\section{Aproximación a las alineaciones astronómicas de las principales edifi- caciones del valle de Jesús de Otoro con respecto a las salidas y puestas de sol en el solsticio de verano e invierno}

Desde la aparición del hombre en la Tierra, este se ha interesado en los fenómenos astronómicos debido, quizá, a la poca contaminación atmosférica, la inexistencia de la iluminación artificial y a la poca distracción que la actualidad ofrece. Poco a poco observó y documentó ciclos que relacionó con el tiempo y la ocurrencia de fenómenos naturales vinculados, por ejemplo con el clima, y se percató de que podía utilizar esta relación de los astros con la naturaleza como guía para marcar estos eventos en su beneficio; a falta de escritura utilizó marcas físicas en el suelo o la combinación con marcas naturales en el horizonte que apuntan hacia un objeto astronómico determinado.

\section{Relación de la astronomía con las culturas pasadas}

La astronomía sigue presente con el nacimiento de las sociedades y la planeación de sus ciudades, un ejemplo clave es la ciudad de Teotihuacán, que se localiza al noreste de la ciudad de México, aproximadamente a 40 kilómetros. Esta orienta su eje principal o Avenida de los Muertos, desviándolo $15^{\circ} 28^{\prime} \mathrm{E}$ desde el norte y su eje perpendicular y secundario orientado hacia $15^{\circ} 21^{\prime} \mathrm{N}$ hacia el oeste. La razón de esta disposición obligó a los constructores teotihuacanos a modificar la topografía en lugar de adaptarse a ella, es la orientación de su eje principal hacia Las Pléyades, pues este grupo de estrellas pasaba por su orto heliaco el mismo día que el primero de los dos pasos del Sol por el cenit, día de mucha importancia en la demarcación de las estaciones, dispositivo ideal para medir el tiempo mediante el Sol y las estrellas (Aveni, 1981).

Así como en Teotihuacán y su alineamiento con Las Pléyades, entre otros, diferentes culturas mesoamericanas en distintas latitudes se probaron con los alineamientos. Pineda de Carías, Véliz y Agurcia Fasquelle (2002), mencionan que en Honduras, la ciudad maya de Copán muestra varios de estos, relacionados con las estaciones y con la medida del tiempo; sin embargo, aún existen miles de sitios arqueológicos inexplorados en esta rama del saber arqueoastronómico, tal es el caso de Jesús de Otoro, en donde llama la atención el urbanismo de sus poblados en forma de C (Cruz Castillo, 2004). 


\section{OBJETIVO}

Ubicar sitios arqueológicos revisando la literatura arqueológica y comprobar en el campo las ubicaciones de los sitios arqueológicos que se encuentren en el valle de Otoro y determinar posibles alineamientos astronómicos con respecto a la posición del Sol en determinadas épocas del año.

\section{METODOLOGÍA}

En esta primera fase del proyecto se registraron todos los sitios arqueológicos que se pudieron encontrar en la literatura arqueológica sobre el valle de Otoro. Posteriormente, se realizó la gira de campo para ubicar cada sitio arqueológico.

La mejor época en Honduras para hacer recorridos o reconocimientos arqueológicos en el campo es durante la época seca, dado que permite movilidad y visibilidad óptimas, porque los matorrales están secos y los arbustos tienen poco follaje, de esta manera es posible movilizarse y reconocer más rápidamente los restos arqueológicos arquitectónicos en la superficie terrestre.

Para la primera visita se llevaron lápices, cuadernos, papel, cámara fotográfica, un GPS, los planos existentes de los sitios arqueológicos y un mapa de la zona con curvas a nivel. El material que se menciona sirvió para tomar notas, hacer croquis que se consideraron necesarios en el momento de la visita al sitio y un registro fotográfico del sitio. Los planos disponibles de los sitios se aprovecharon para ubicarse en el sitio y reconocer mejor las estructuras.

Aprovechando la información obtenida por medio de revisiones bibliográficas, la que proporcionaron algunos vecinos de la zona de estudio y con la asistencia del guía contratado, el equipo de campo conformado por tres investigadores, se movilizó en vehículo por el valle. Las tareas para este equipo fueron las siguientes: uno de los investigadores se encargó del aspecto logístico, la movilización en el campo a cada sitio, poniéndose de acuerdo con el guía y el regreso a los sitios en caso de necesidad para hacer alguna comprobación; otro investigador se encargó del manejo del GPS, asegurando lecturas apropiadas y confiables sobre la ubicación de cada sitio; el tercer investigador fue el encargado de registrar la información general o de las notas pertinentes para cada sitio y registrar las lecturas del GPS que proporcionó el encargado de este instrumento.

De regreso a la Ciudad Universitaria, siguió la revisión, estudio y análisis de toda la información bibliográfica y de campo recopilada. Con el personal capacitado en los 
sistemas de información geográfica (SIG), se buscó la imagen satelital que cubre la zona del valle de Otoro y ahí se registró la posición de cada sitio arqueológico. De igual manera se realizaron análisis relacionados con la preservación y conservación de los sitios arqueológicos en el valle, tales como vulnerabilidad por inundaciones, deslizamientos y amenazas antropogénicas (Ver figura 1).

Figura 1. Detalle de amenazas a sitios arqueológicos en el municipio de Jesús de Otoro, Valle de Otoro, Intibucá*
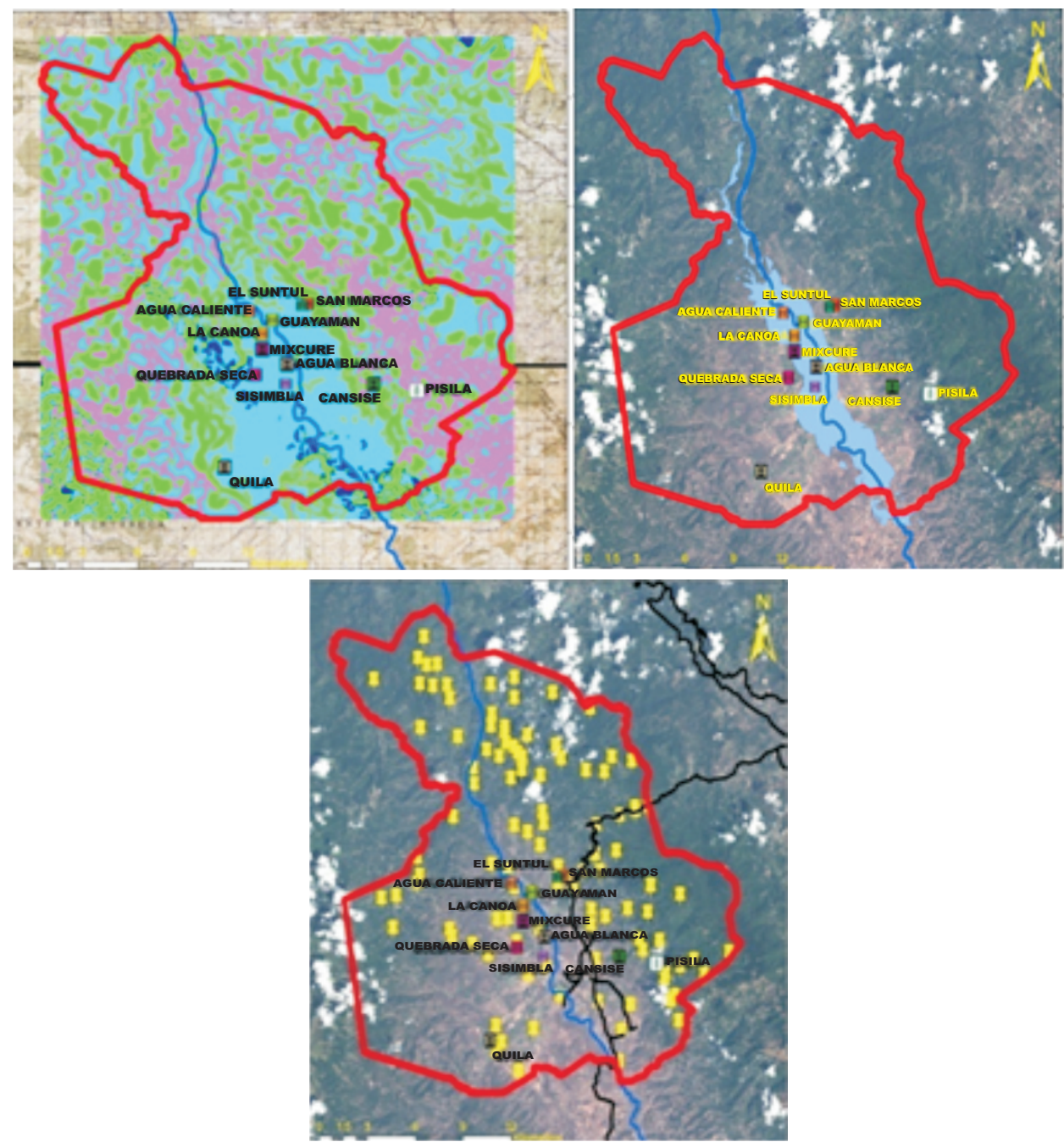

Fuente: Elaboración propia, datos SINIT.

*Vulnerabilidad al deslizamiento (izquierda), inundación (centro) y crecimiento urbano (derecha). 
Una vez que se logró la ubicación en los SIG, se buscó el significado arqueoastronómico de cada sitio, es decir, se trató de encontrar la relación de cada sitio con algún astro, en especial con el Sol. En vista de que el valle es pequeño, también se trató de ver si a todos los sitios arqueológicos, como un conjunto, se les puede asignar alguna interpretación arqueoastronómica.

El instrumento principal para la toma de datos fue el GPS Mobile Mapper 6, marca Magellan, con un margen de error de apenas 1 metro de diámetro por toma de punto. Se configuró con un tiempo de exposición de sesenta segundos para la toma de cada uno de los puntos.

El procedimiento de trabajo consistió en tomar puntos en el centro de las plazas principales de cada sitio arqueológico y en cada esquina de la estructura principal. Además, se visitó y se tomaron datos de un sitio con arte rupestre para poder buscar probables alineamientos con los demás sitios arqueológicos de la zona. Los datos en cuestión se han cotejado y determinado posibles alineamientos astronómicos.

Para la realización del proyecto se planteó la adquisición de equipo y una gira de campo para la recolección de datos en cada uno de los sitios arqueológicos de la zona. La adquisición del equipo básicamente consistió en la adquisición de un GPS de alta precisión, equipo de oficina y presupuesto para el alquiler de un vehículo $4 \times 4$, viáticos para el personal del Departamento y el pago de un guía local para el recorrido.

\section{MUESTREODE CAMPO}

La mayoría de los sitios arqueológicos en el municipio de Jesús de Otoro, valle de Otoro, se encuentran en la parte central del valle, distribuidos de la siguiente manera: en la margen izquierda del río Grande de Otoro, se encuentran nueve (9) sitios arqueológicos. Por esta margen, el sitio más al sur es Quila y el de más al norte es Quebrada Seca. Todos los sitios están entre la Quebrada de Otoro, por el sur, y el río Siruna por el norte.

Por la margen derecha, además del pueblo de Jesús de Otoro, hay cuatro (4) sitios arqueológicos. El sitio más al sur es Pisila y el de más al norte es San Marcos. Todos los sitios están entre la quebrada San Miguel, al sur, y la quebrada San Marcos al norte; exceptuando Pisila, que está a unos 1,550 msnm. Todos los sitios en el valle están a menos de 700 msnm (Ver figura2).

Además del informe bastante completo del trabajo de campo de Cruz Castillo (2004), también se logró información de Inestroza (2007) y de McFarlane y Stockett 
(2006). Estos datos bibliográficos permitieron apoyar y corroborar nuestro trabajo durante la visita al área de estudio.

La visita al valle de Otoro se extendió por cuatro días únicamente, incluyendo el tiempo de viaje de ida y de regreso, lo que abarcó aproximadamente unas cuatro horas y media de esos cuatro días. En algunos de estos días fue necesario cortar el trabajo de campo temprano, debido a las lluvias. La gira tuvo el siguiente itinerario:

- 29 de mayo:Agua Caliente y Quebrada Seca

- 30 de mayo: Sinsimbla, Agua Blanca, Mixcure, La Canoa y Tatumbla

- 31 de mayo: San Marcos, Guayamán, EI Suntul, Cancise y Quila

- 1 de junio: Pisila (Verfiguras 3 y 4)

Figura 2. Detalle de la ubicación de los sitios arqueológicos registrados en el municipio de Jesús de Otoro, valle de Otoro, Intibucá

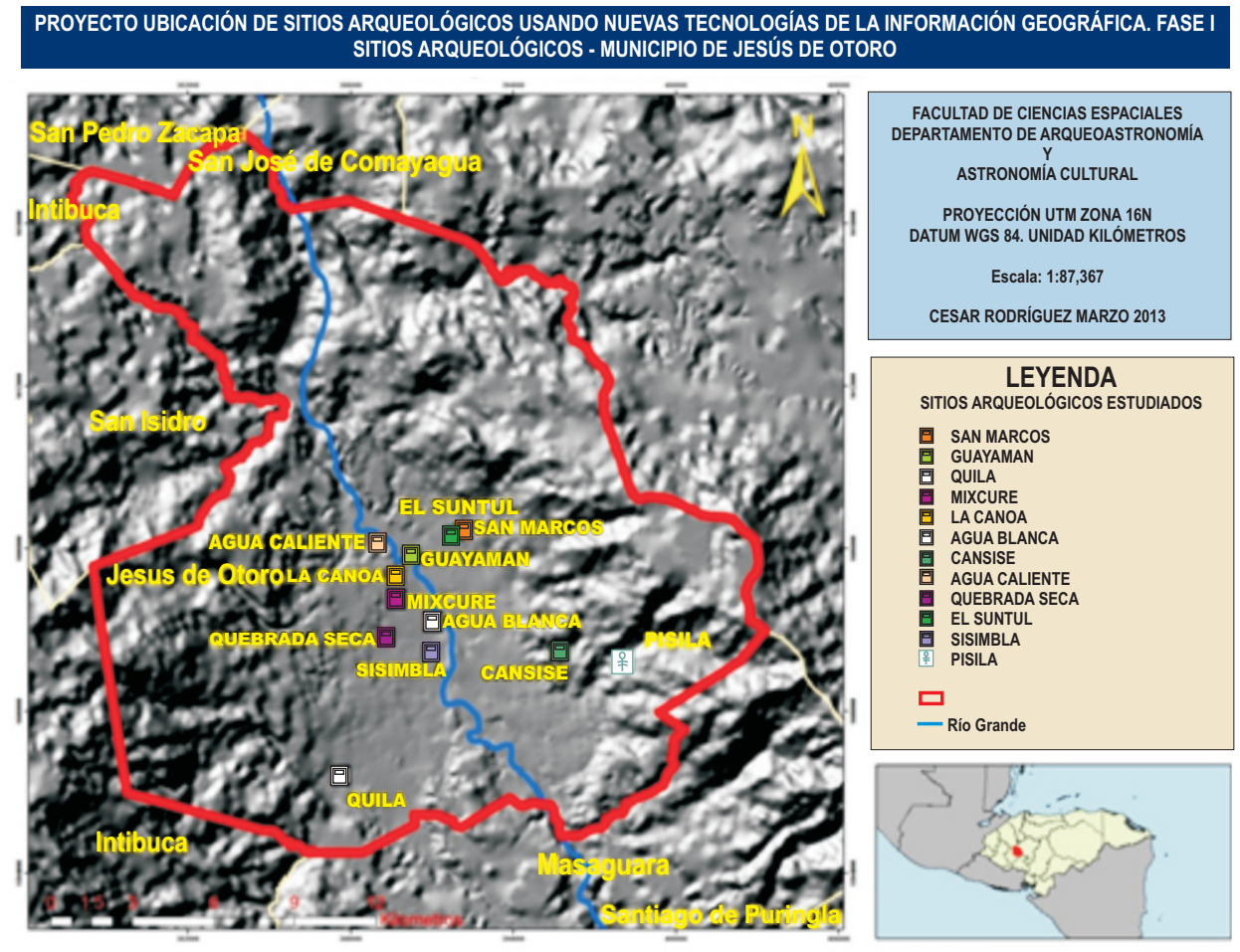

Fuente: Elaboración propia, datos SINIT 
Figura 3. Sitio San Marcos

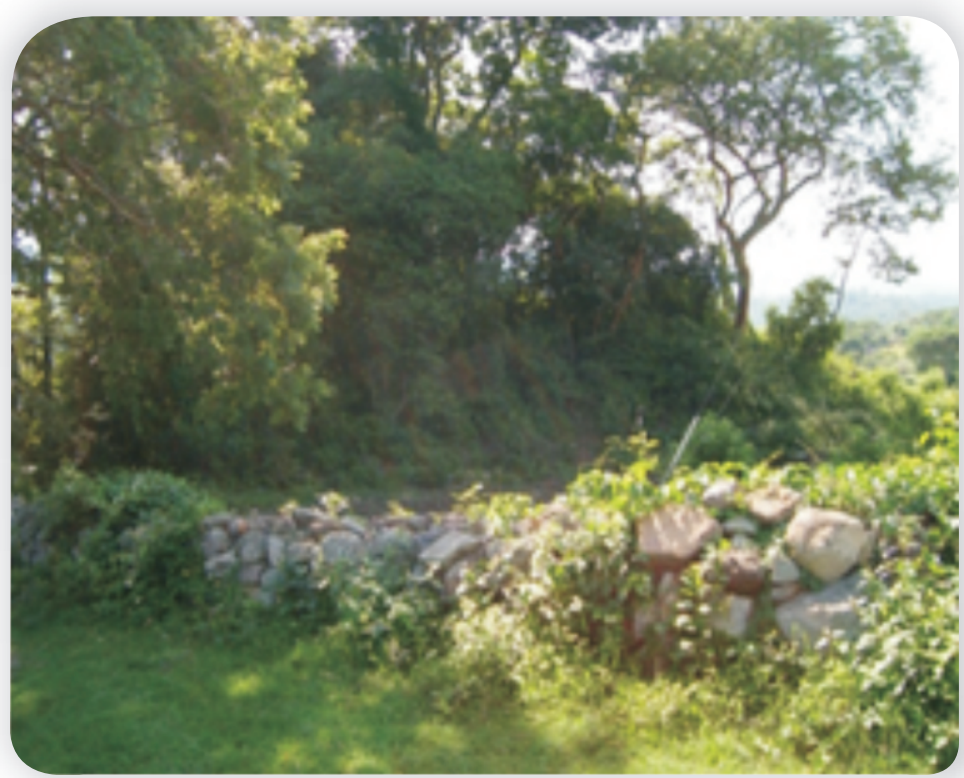

Estructura principal de unos 16 metros de altura, obsérvese el canto de río de la estructura utilizado para la elaboración de un cerco perimetral.

Figura 4. Estructura principal de Guayamán (G-1)

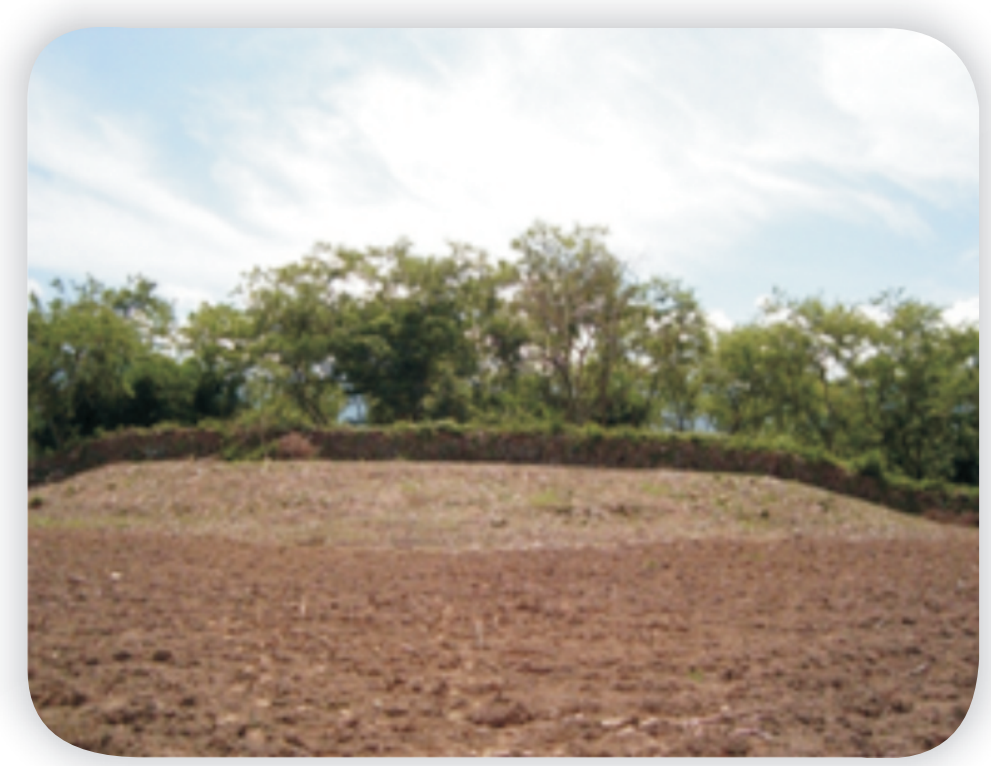

Al igual que en San Marcos, el canto de río de las estructuras fue utilizado para la elaboración de un cerco perimetral. 


\section{Astronomía de posición}

En la búsqueda de alineamientos en arqueoastronomía es preciso encontrar la ubicación en la esfera celeste de un astro en particular, para relacionarlo con un objeto en la Tierra, esta posición se determina mediante coordenadas polares 0 esféricas, pues están ubicados los astros sobre una esfera celeste de radio imaginario en donde las distancias son arcos y no rectas.

Para las coordenadas esféricas es necesario definir algunas referencias, por ejemplo un círculo básico máximo; uno horizontal, el ecuador celeste, que es la proyección del ecuador terrestre para las declinaciones y a partir del cual se trazarán otros secundarios paralelos y otros verticales a él. Perpendicular al plano de este círculo se traza una vertical que corta la esfera en dos puntos, los polos, y que marca el punto de origen de las coordenadas.

El sistema de coordenadas celestes que se adapta a la búsqueda de alineaciones por medio de coordenadas geográficas encontradas mediante GPS es el altacimutal o sistema de coordenadas horizontales, que es un sistema de coordenadas topo céntrico horizontal, con punto de referencia en el lugar geográfico del observador sobre la superficie terrestre. Las coordenadas horizontales serán, entonces, el acimut y la altura (Contell, et al, 2006).

\section{RESULTADOS: ANÁLISIS DE DATOS, CARTOGRAFÍA Y ALINEAMIENTOS}

\section{Uso de sistemas de información geografía (SIG)}

Primero se definió la ubicación geográfica y categorías arqueológicas de los sitios investigados, específicamente en los límites del municipio de Jesús de Otoro, departamento de Intibucá. Sobresale la gran cantidad de sitios de categoría principal (5) distribuidos en el área de estudio, acompañados de sitios arqueológicos con categorías 3 y 4, así como petrograbados. Lo anterior demuestra la gran importancia social y económica del área en la antigüedad y da razones suficientes para realizar estudios futuros (arqueológicos, antropológicos, arqueoastronómicos); en consecuencia, por ahora es importante velar por su conservación.

Es importante considerar cómo está cambiando la cobertura de tierra de la cuenca del valle. En la investigación: Análisis multitemporal de la cobertura de la tierra en la cuenca del valle de Jesús de Otoro entre los años 2000-2006 (Sánchez, 2009), se demuestra el avance agrícola sobre el área entre los años, sobre todo por guamiles y café, lo que aumenta la presión para los yacimientos arqueológicos, especialmente 
porque la mayoría se encuentra cerca de zonas pobladas e intervenidas por cultivos, lo que pone en peligro su existencia ante el avance de las actividades antropogénicas en la zona.

Esto se comprueba al revisar las imágenes que se presentan en el presente estudio de los diferentes yacimientos arqueológicos estudiados, ya que se notó en los sitios la gravedad de la situación durante la visita de campo hecha por el equipo de investigación del proyecto (Ver figura 5).

Figura 5. Detalle de los sitios arqueológicos registrados en el municipio de Jesús de Otoro, valle de Otoro, Intibucá y su relación con la cobertura del suelo

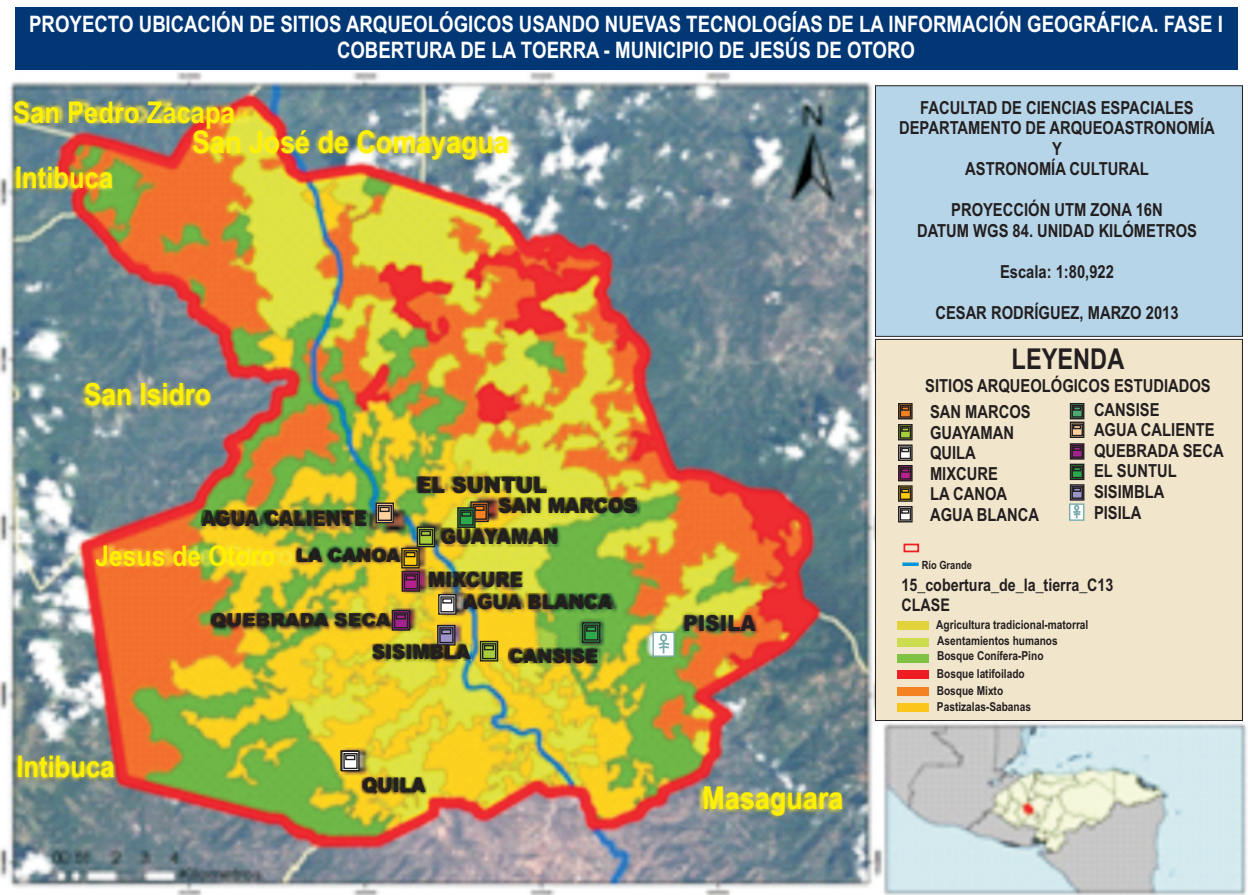

Fuente: Elaboración propia, datos SINIT.

Sobre estos sitios, McFarlane y Stockett (2007) señalan que: "En general, son conservados muy pobremente; el saqueo en las estructuras grandes es común y la mayoría de las estructuras no monumentales fueron destruidas para crear campos de agricultura".

Lo anterior condujo a elaborar, además de la cartografía de ubicación de los sitios 
arqueológicos en el aérea de estudio y sus categorías arqueológicas, cartografía adicional en la cual se presenta la vulnerabilidad por amenazas (antropogénicas y naturales) a la conservación del patrimonio cultural precolombino en esta zona.

Se presentan mapas de vulnerabilidad por centros poblados alrededor de los yacimientos arqueológicos, por riesgo de inundaciones y deslizamientos. Se evidencia que la mayoría de los sitios investigados son vulnerables a todas 0 alguna de las amenazas a su conservación. Imágenes y fotografías aéreas de la zona de estudio enriquecen la base de datos de la investigación, estando disponibles para una segunda fase de investigación (Ver figura 6).

Figura 6. Detalle de los sitios arqueológicos registrados en el municipio de Jesús de Otoro, valle de de Otoro, Intibucá y su clasificación arqueológica

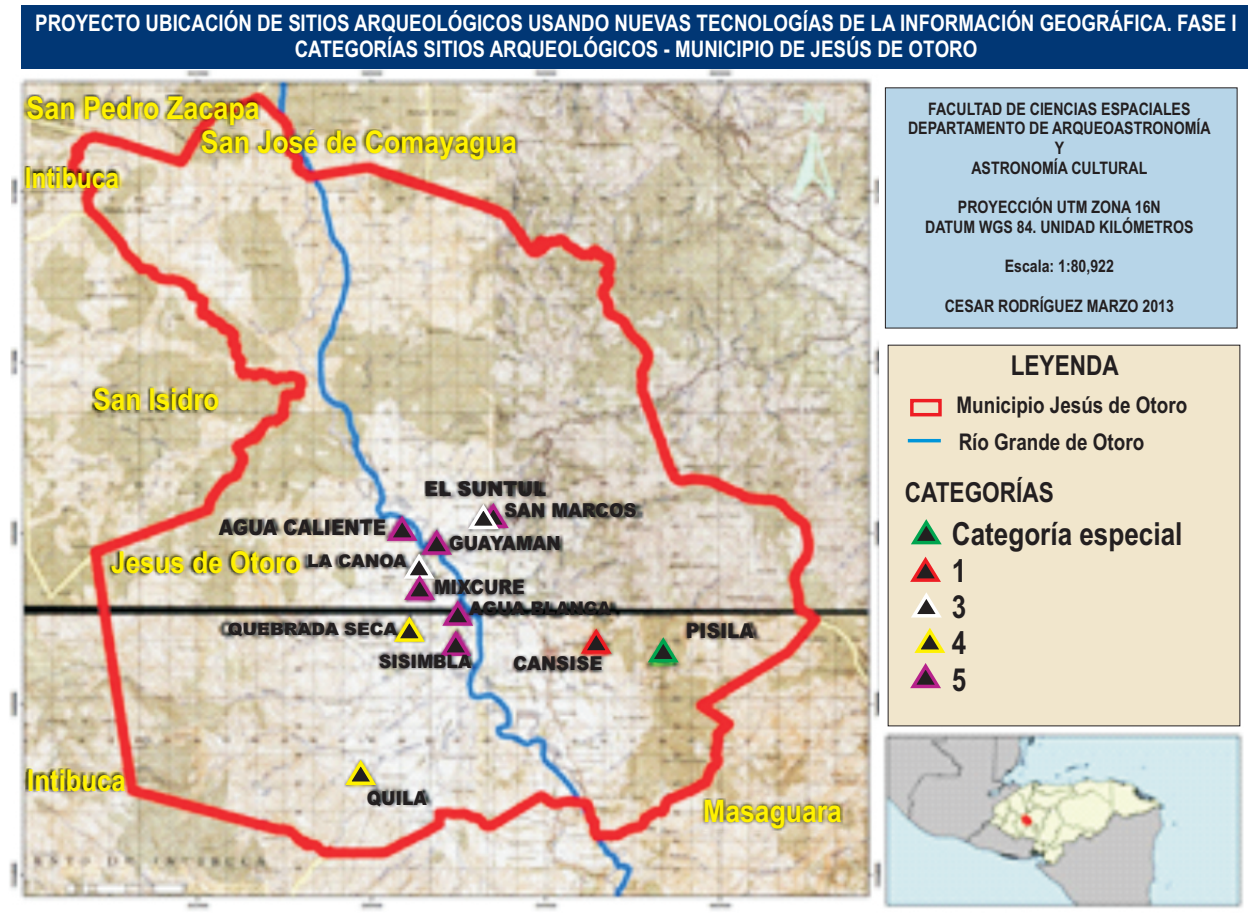

Fuente: Elaboración propia, datos SINIT.

Categorías: 5 en morado, 4 en amarillo, 3 en blanco, 1 en rojo y Categoría especial en verde.

Los cuadros 1, 2 y 3 resumen los análisis de los datos de campo referentes a los sitios arqueológicos del valle de Otoro, estudiados durante la investigación y aportes de otras investigaciones relacionadas. 
Cuadro 1. Lista descendente de los sitios arqueológicos del valle, según su categoría

\begin{tabular}{|c|l|c|c|}
\hline No. & Sitio arqueológico & Categoría & Total \\
\hline 1 & Agua Blanca & 5 & \\
\hline 2 & Mixcure & 5 & \\
\hline 3 & Sisimbla & 5 & 6 \\
\hline 4 & Agua Caliente & 5 & \\
\hline 5 & San Marcos & 5 & \\
\hline 6 & Guayamán & 5 & 2 \\
\hline 1 & Quila & 4 & \\
\hline 2 & Quebrada Seca & 4 & 3 \\
\hline 1 & Naranjo & 3 & 1 \\
\hline 2 & La Canoa & 3 & 1 \\
\hline 3 & Suntil & 1 posible & \\
\hline 1 & Cancise & Petrograbados & 1 \\
\hline 1 & Pisila & Colonial & 14 \\
\hline 1 & Tatumbla & & \\
\hline
\end{tabular}

Fuente: Elaboración propia.

Cuadro 2. Sitios arqueológicos del valle de Otoro

\begin{tabular}{|c|c|c|c|c|c|c|c|}
\hline \multirow[b]{2}{*}{ No. } & \multirow{2}{*}{$\begin{array}{c}\text { Sitio } \\
\text { arqueológico }\end{array}$} & \multirow{2}{*}{$\begin{array}{c}\text { UTM, según } \\
\text { Cruz } \\
\text { Castillo }\end{array}$} & \multirow{2}{*}{$\begin{array}{c}\text { UTM, según } \\
\text { nuestro } \\
\text { proyecto }\end{array}$} & \multirow{2}{*}{$\begin{array}{c}\text { Margen río } \\
\text { Grande de } \\
\text { Otoro }\end{array}$} & \multicolumn{2}{|c|}{ Orientación } & \multirow{2}{*}{ Categoría } \\
\hline & & & & & Cruz & Veliz & \\
\hline 1 & Quila & No datos & $\begin{array}{c}387638 \\
1597592\end{array}$ & Izquierda & EO & EO & 4 \\
\hline 2 & Tatumbla & $\begin{array}{c}\text { N1601300 } \\
\text { E389500 }\end{array}$ & No datos & Izquierda & NE & NE & Colonial \\
\hline 3 & Sinsimbla & $\begin{array}{c}\text { N1602200 } \\
\text { E391200 }\end{array}$ & $\begin{array}{c}390948 \\
1602205\end{array}$ & Izquierda & NO & NO & 5 \\
\hline 4 & Naranjo & $\begin{array}{c}\mathrm{N} 1602800 \\
\text { E390750 }\end{array}$ & No datos & Izquierda & $\begin{array}{l}\text { No fue } \\
\text { visitado }\end{array}$ & $\begin{array}{l}\text { No fue } \\
\text { visitado }\end{array}$ & 3 \\
\hline 5 & Agua Blanca & No datos & $\begin{array}{c}391013 \\
1603270\end{array}$ & $\begin{array}{l}\text { Izquierda, entre } \\
\text { Q. Mixcure y } \\
\text { Agua Blanca }\end{array}$ & NO & NO & 5 \\
\hline 6 & Mixcure & $\begin{array}{l}\text { N1603300 } \\
\text { E390000 }\end{array}$ & $\begin{array}{c}389674 \\
1604096\end{array}$ & $\begin{array}{l}\text { Izquierda, izq. } \\
\text { Q. Mixcure }\end{array}$ & NO & NO & 5 \\
\hline 7 & La Canoa & $\begin{array}{l}\text { N1604600 } \\
\text { E390650 }\end{array}$ & $\begin{array}{c}389678 \\
1604973\end{array}$ & Izquierda & NO & NO & 3 \\
\hline
\end{tabular}




\begin{tabular}{|c|c|c|c|c|c|c|c|}
\hline \multirow[b]{2}{*}{ No. } & \multirow{2}{*}{$\begin{array}{c}\text { Sitio } \\
\text { arqueológico }\end{array}$} & \multirow{2}{*}{$\begin{array}{c}\text { UTM, según } \\
\text { Cruz } \\
\text { Castillo }\end{array}$} & \multirow{2}{*}{$\begin{array}{c}\text { UTM, según } \\
\text { nuestro } \\
\text { proyecto }\end{array}$} & \multirow{2}{*}{$\begin{array}{c}\text { Margen río } \\
\text { Grande de } \\
\text { Otoro }\end{array}$} & \multicolumn{2}{|c|}{ Orientación } & \multirow{2}{*}{ Categoría } \\
\hline & & & & & Cruz & Veliz & \\
\hline 8 & Agua Caliente & $\begin{array}{c}\text { N1606650 } \\
\text { E388980 }\end{array}$ & $\begin{array}{c}389002 \\
1606224\end{array}$ & Izquierda & NNO & NNO & 5 \\
\hline 9 & $\begin{array}{l}\text { Quebrada } \\
\text { Seca }\end{array}$ & $\begin{array}{c}\text { N1606950 } \\
\text { E388150 }\end{array}$ & $\begin{array}{c}389308 \\
1602703\end{array}$ & $\begin{array}{l}\text { Izquierda, } \\
\text { izq. Q. seca }\end{array}$ & NNO & NNO & 4 \\
\hline 10 & San Marcos & $\begin{array}{c}N 1606550 \\
\text { E392050 }\end{array}$ & $\begin{array}{c}392163 \\
1606645\end{array}$ & $\begin{array}{l}\text { Derecha, izq. } \\
\text { Q. San Marcos }\end{array}$ & NE & NE & 5 \\
\hline 11 & Suntul & $\begin{array}{c}\text { N1605900 } \\
\text { E391400 }\end{array}$ & $\begin{array}{c}391865 \\
1606559\end{array}$ & $\begin{array}{l}\text { Derecha, izq. } \\
\text { Q. San Marcos }\end{array}$ & NO & NO & 3 \\
\hline 12 & Guayamán & No datos & $\begin{array}{c}390242 \\
1605738\end{array}$ & $\begin{array}{l}\text { Derecha, izq. } \\
\text { Q. San Marcos }\end{array}$ & - & NE & 5 \\
\hline 13 & Cansise & No datos & $\begin{array}{c}395707 \\
1602193\end{array}$ & Derecha & - & - & 1 posible \\
\hline 14 & Pisila & No datos & $\begin{array}{c}398040 \\
1601818\end{array}$ & $\begin{array}{l}\text { Derecha, izq. } \\
\text { Q. Santa Cruz }\end{array}$ & NO & NO & Especial \\
\hline
\end{tabular}

Fuente: Elaboración propia.

Cuadro 3. Análisis de las coberturas de tierra de la cuenca del valle de de Otoro, 2000-2006

\begin{tabular}{|c|c|c|c|c|c|}
\hline \multirow{2}{*}{ Coberturas } & \multicolumn{2}{|c|}{ Clasificación año 2000} & \multicolumn{2}{|c|}{ Clasificación año 2006} & \multirow{2}{*}{ Diferencias } \\
\hline & Área (ha) & $\%$ & Área (ha) & $\%$ & \\
\hline Bosque latifoliado & 23,742 & 13.53 & 24,173 & 13.78 & +431 \\
\hline Bosque coníferas denso & 24,893 & 14.19 & 23,803 & 13.57 & $-1,090$ \\
\hline Bosque coníferas ralo & 13,460 & 7.67 & 16,560 & 9.44 & $+3,100$ \\
\hline Guamiles o café & 36,988 & 21.8 & 39,098 & 22.28 & $+2,110$ \\
\hline Matorral & 22,855 & 13.03 & 25,156 & 14.34 & $+2,301$ \\
\hline Arbustos-pastos o cultivos & 53,028 & 30.22 & 46,211 & 26.34 & $-6,817$ \\
\hline $\begin{array}{l}\text { Urbano o suelos } \\
\text { desnudos }\end{array}$ & 240 & 0.14 & 205 & 0.12 & -35 \\
\hline Cuerpos de agua & 224 & 0.13 & 224 & 0.13 & 0 \\
\hline Totales & 175,430 & 100 & 175,430 & 100 & 00.00 \\
\hline
\end{tabular}

Fuente: Sánchez, 2009

\section{Alineaciones astronómicas de las principales edificaciones del valle de Otoro con respecto a las salidas y puestas de Sol en el solsticio de verano e invierno}

Al revisar estrictamente los resultados, en su mayoría no se encontró relación exacta de alineamientos con las orientaciones de las estructuras de los diferentes sitios del valle de Otoro con eventos del Sol, como el solsticio de invierno o de 
verano, a excepción del lado 3-2 y 2-3 del sitio Guayamán A (base de la estructura), cuyo error es $0^{\circ}$ y coinciden con SSV y PSI satisfactoriamente.

Como se observa en el cuadro 4, resaltan los recuadros de la columna de valores absolutos menores con valores de $1.08^{\circ}$ hasta $6.02^{\circ}$. Los sitios que tienen suficientes datos para comparar y al menos uno de los lados de la estructura se aproxima al acimut del Sol en la SSV y PSI con una diferencia cercana a los cinco grados, en los que posiblemente exista una relación de orientación al calcular el acimut tomando en cuenta el valor real de la altura del horizonte para cada uno de esos sitios arqueológicos, como el caso de San Marcos que muestra aproximaciones en alineamientos por los valores cercanos a cero, sin embargo, el lado 2-3 (5.41 a $5.88^{\circ}$ de acimut) y el lado $4-5$ ( $1.55^{\circ}$ a 1.08 de acimut) por sus valores se muestra que no son lados paralelos; en el resto de lados de las estructuras la diferencia observada en la diferencia de acimut es mucho mayor.

Los sitios que tienen suficientes datos para comparar y al menos uno de los lados de la estructura se aproxima al acimut del Sol en la SSV y PSI con una diferencia cercana a los cinco grados. En el resto de lados de las estructuras la diferencia observada es mucho mayor.

\section{Acimut de las estructuras y del Sol}

Para cada sitio arqueológico del valle de Otoro se eligió la estructura más representativa del grupo en cuanto a dimensiones, tomando las coordenadas geográficas de los vértices de las bases de las edificaciones seleccionadas para luego convertirlas a latitud y longitud. Fue necesaria la depuración de coordenadas eligiendo las estructuras de los sitios de los que se tenían datos precisos y suficientes que permitieran realizar el análisis arqueoastronómico con respecto a los solsticios de verano e invierno (Ver cuadro 4). 
Cuadro 4. Coordenadas geográficas y ángulos acimutales de los sitios del valle de Jesús de Otoro y ortos y ocasos solsticiales solares

\begin{tabular}{|c|c|c|c|c|c|c|c|}
\hline \multicolumn{8}{|c|}{ Aplicación de las fórmulas para alineamientos en el valle de Otoro } \\
\hline \multirow[t]{2}{*}{$\begin{array}{c}\text { Sitiol } \\
\text { estructura }\end{array}$} & \multirow[t]{2}{*}{$\begin{array}{l}\text { Lado de la } \\
\text { estructura }\end{array}$} & \multicolumn{2}{|c|}{$\begin{array}{c}\text { Fuente: Bertilio Amaya, } \\
\text { GPS Mobile Mapper 6, } \\
\text { marca Magellan }\end{array}$} & \multirow{2}{*}{$\begin{array}{l}\text { Acimut para } \\
\text { cada lado } \\
\text { de la estruc- } \\
\text { tura }\end{array}$} & \multirow[t]{2}{*}{ * } & \multirow[t]{2}{*}{$\begin{array}{l}\text { Acimut } \\
\text { sol }\end{array}$} & \multirow{2}{*}{$\begin{array}{l}\text { Valor absoluto de } \\
\text { la diferencia en } \\
\text { grados entre la } \\
\text { orientación de los } \\
\text { puntos y el ángulo } \\
\text { de referencia }\end{array}$} \\
\hline & & Latitud & Longitud & & & & \\
\hline \multirow[t]{8}{*}{ La Canoa } & $\begin{array}{l}\text { Acimut } 3 \\
\text { desde 2= }\end{array}$ & \multirow[t]{2}{*}{$\begin{array}{l}14.5158411 \\
1\end{array}$} & \multirow[t]{2}{*}{88.01881222} & 70.37465867 & SSV & 65.490353 & 4.88 \\
\hline & $\begin{array}{l}\text { Acimut } 2 \\
\text { desde 3= }\end{array}$ & & & 250.3747277 & PSI & 245.963217 & 4.41 \\
\hline & $\begin{array}{l}\text { Acimut } 4 \\
\text { desde 3= }\end{array}$ & \multirow[t]{2}{*}{$\begin{array}{l}14.5159361 \\
1\end{array}$} & \multirow[t]{2}{*}{88.01853703} & 156.7580662 & SSI & 114.036783 & 42.72 \\
\hline & $\begin{array}{l}\text { Acimut } 3 \\
\text { desde 4= }\end{array}$ & & & 336.7580885 & PSV & 294.509647 & 42.25 \\
\hline & $\begin{array}{l}\text { Acimut } 5 \\
\text { desde4= }\end{array}$ & \multirow[t]{2}{*}{$\begin{array}{l}14.5157355 \\
6\end{array}$} & \multirow[t]{2}{*}{88.01844806} & 260.1251204 & PSI & 245.963217 & 14.16 \\
\hline & $\begin{array}{l}\text { Acimut } 4 \\
\text { desde } 5=\end{array}$ & & & 80.12505304 & SSV & 65.490353 & 14.63 \\
\hline & $\begin{array}{l}\text { Acimut } 5 \\
\text { desde 2= }\end{array}$ & \multirow[t]{2}{*}{$\begin{array}{l}14.5156902 \\
8\end{array}$} & \multirow[t]{2}{*}{88.01871667} & 148.4791877 & SSI & 114.036783 & 34.44 \\
\hline & $\begin{array}{l}\text { Acimut } 2 \\
\text { desde 5= }\end{array}$ & & & 328.4792116 & PSV & 294.509647 & 33.97 \\
\hline \multirow[t]{8}{*}{ Guayamán A } & $\begin{array}{l}\text { Acimut } 2 \\
\text { desde 1= }\end{array}$ & \multirow[t]{2}{*}{$\begin{array}{l}14.5225861 \\
1\end{array}$} & \multirow[t]{2}{*}{88.01826861} & 153.514768 & SSI & 114.038441 & 39.48 \\
\hline & $\begin{array}{l}\text { Acimut } 1 \\
\text { desde 2= }\end{array}$ & & & 333.514807 & PSV & 294.511880 & 39.00 \\
\hline & $\begin{array}{l}\text { Acimut } 3 \\
\text { desde 2= }\end{array}$ & \multirow[t]{2}{*}{$\begin{array}{l}14.5222838 \\
9\end{array}$} & \multirow[t]{2}{*}{88.01811306} & 65.9589202 & SSV & 65.48120 & 0.48 \\
\hline & $\begin{array}{l}\text { Acimut } 2 \\
\text { desde } 3=\end{array}$ & & & 245.959012 & PSI & 245.961559 & 0.00 \\
\hline & $\begin{array}{l}\text { Acimut } 4 \\
\text { desde } 3=\end{array}$ & \multirow[t]{2}{*}{$\begin{array}{l}14.5224413 \\
9\end{array}$} & \multirow[t]{2}{*}{88.01774833} & 327.250137 & SSV & 65.488120 & 261.76 \\
\hline & $\begin{array}{l}\text { Acimut } 3 \\
\text { desde 4= }\end{array}$ & & & 147.250089 & SSV & 114.038441 & 33.21 \\
\hline & $\begin{array}{l}\text { Acimut } 4 \\
\text { desde 1= }\end{array}$ & \multirow[t]{2}{*}{$\begin{array}{l}14.5227336 \\
1\end{array}$} & \multirow[t]{2}{*}{88.01794250} & 64.956399 & SSI & 245.961559 & -181.01 \\
\hline & $\begin{array}{l}\text { Acimut } 4 \\
\text { desde 1= }\end{array}$ & & & 244.956481 & PSI & 65.488120 & 179.47 \\
\hline \multirow[t]{8}{*}{ Guayamán B } & $\begin{array}{l}\text { Acimut } 7 \\
\text { desde 6= }\end{array}$ & \multirow[t]{2}{*}{$\begin{array}{l}14.5225427 \\
8\end{array}$} & \multirow[t]{2}{*}{88.01810361} & 139.144263 & SSV & 114.038441 & 25.11 \\
\hline & $\begin{array}{l}\text { Acimut } 6 \\
\text { desde } 7=\end{array}$ & & & 319.14429 & SSI & 294.511880 & 24.63 \\
\hline & $\begin{array}{l}\text { Acimut } 8 \\
\text { desde } 7=\end{array}$ & \multirow[t]{2}{*}{$\begin{array}{l}14.5224202 \\
8\end{array}$} & \multirow[t]{2}{*}{88.01799417} & 71.5040517 & PSV & 65.488120 & 6.02 \\
\hline & $\begin{array}{l}\text { Acimut } 7 \\
\text { desde } 8=\end{array}$ & & & 251.504086 & PSI & 245.961559 & 5.54 \\
\hline & $\begin{array}{l}\text { Acimut } 5 \\
\text { desde } 8=\end{array}$ & \multirow[t]{2}{*}{$\begin{array}{l}14.5225861 \\
1\end{array}$} & 88.01826861 & 309.649294 & PSV & 294.511880 & 15.14 \\
\hline & $\begin{array}{l}\text { Acimut } 8 \\
\text { desde } 5=\end{array}$ & & & 129.649257 & SSI & 114.03844 & 15.61 \\
\hline & $\begin{array}{l}\text { Acimut } 6 \\
\text { desde } 5=\end{array}$ & $\begin{array}{l}14.5222838 \\
9\end{array}$ & 88.01811306 & 66.3091294 & PSI & 245.961559 & -179.65 \\
\hline & $\begin{array}{l}\text { Acimut } 5 \\
\text { desde } 6=\end{array}$ & & & 246.309154 & SSV & 65.488120 & 180.82 \\
\hline
\end{tabular}




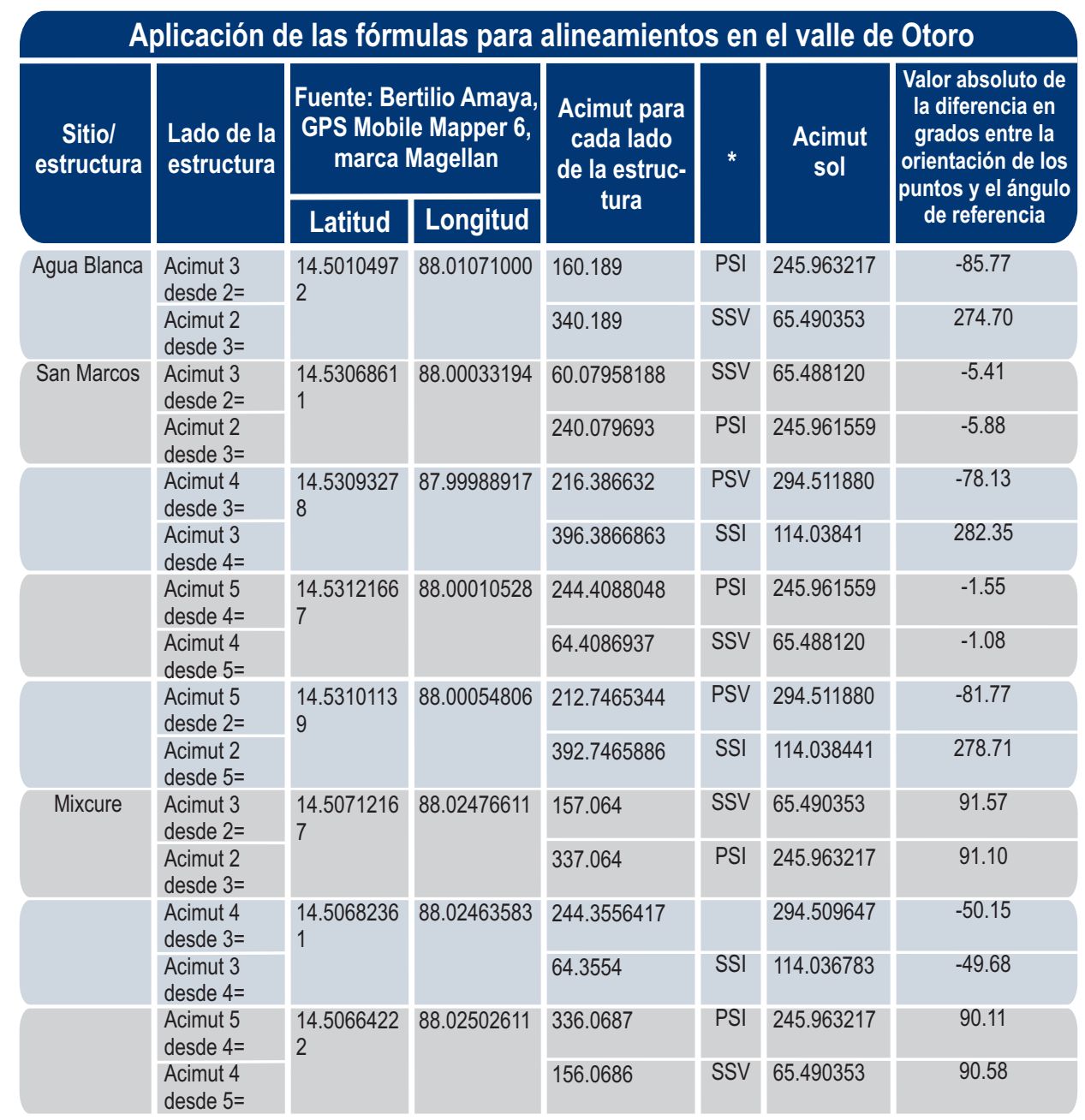

Fuente: Elaboración propia.

Aparecen los cálculos de la salida del Sol en el solsticio de verano (SSV), puesta del Sol en el solsticio de invierno (PSI), puesta del Sol en el solsticio de verano (PSV) y la salida del Sol en el solsticio de invierno (SSI) y la columna en la que se lee la diferencia entre la orientación de los puntos por cada lado de la edificación y el ángulo de referencia 0 acimut del Sol que determinará la posibilidad de alineamientos con los eventos solares ya mencionados. 


\section{CONCLUSIONES GENERALES}

1. En general, todos los sitios visitados en esta gira muestran características similares que van de acuerdo con su categoría, es decir, los sitios categoría 5 muestran casi los mismos patrones (estructuras montadas en plataformas grandes de forma piramidal, elaboradas con canto de río, con un patio principal rodeado de estructuras menores, alineadas por lo general noreste o noroeste y con un patrón en forma de $\mathrm{C}$ y así sucesivamente. Llama poderosamente la atención la concentración de los sitios arqueológicos en el valle, lo que sugiere que en algún período de su cronología pudo haber existido una gran riqueza, tal como se nota en la actualidad con la actividad agrícola y ganadera; consecuentemente, todavía falta mucha investigación de índole arqueológico, etnohistórico e histórico por realizarse en el lugar, desde el campo de la arqueoastronomía se brinda este acercamiento al saber de estos antiguos pueblos que habitaron dicho valle, lo que puede ser la punta de la lanza hacia el resto de los campos del saber anteriormente mencionados.

2. Se espera que los resultados sirvan de apoyo a los docentes del Departamento de Arqueoastronomía y Astronomía Cultural en la impartición de la asignatura optativa: Introducción a la Arqueoastronomía. De esa manera, tanto profesores como estudiantes se estarán beneficiando y compartiendo conocimientos recientes sobre Arqueología, Arqueoastronomía y las técnicas más recientes que se aprovechan en estos campos del saber.

3. Es por tal razón que el objetivo era ubicar sitios arqueológicos en hojas cartográficas y en SIG. En una etapa posterior de este proyecto, se aprovechará la información sobre la ubicación de estos sitios arqueológicos para analizar las imágenes satelitales que cubren distintas zonas y encontrar las características de los pixeles que representan a esos sitios arqueológicos.

4. La relación puede ser entre monumentos de un sitio arqueológico y sitios arqueológicos. La ubicación exacta de los distintos sitios arqueológicos y los planos exactos de esos sitios pueden ayudar a encontrar más fácilmente y con mayor precisión esas relaciones.

5. Si algunos de los objetivos de la Arqueoastronomía son comprobar que nuestros antepasados tuvieron razones para ubicar y orientar sus obras arquitectónicas tal como lo hicieron, conocer la relación de monumentos entre sí, con los astros y también la relación entre sitios arqueológicos con esos mismos astros, entonces es necesario conocer tanto la ubicación como la orientación exactas de esos monumentos y de esos sitios arqueológicos.

6. Con los resultados también se estará contribuyendo con el Instituto Hondureño de Antropología e Historia ( $\mathrm{HAH})$ en la conformación del atlas arqueológico, 
proyecto en ejecución desde hace ya varios años. El IHAH es la institución gubernamental encargada y responsable de todos los bienes culturales del Estado y con quien tiene que hacerse los arreglos pertinentes para poder trabajar en cualquier zona, sitio arqueológico o cultural.

7. Está muy comprobado que al turista moderno le interesa mucho la naturaleza, además de lo cultural; razón por la cual en esta investigación se proyectan ambos aspectos. Por un lado, está la parte natural que se refleja en el conocimiento y aprovechamiento de los astros y, por otro, tenemos los restos culturales cuyo significado completo se profundiza buscando y demostrando su relación con los astros.

8. En uno de los sitios, Guayamán, hay una sutil relación de los lados de las estructuras con la salida del Sol en el solsticio de verano y con la puesta del Sol en el solsticio de invierno; de manera que posiblemente los emplazamientos de las estructuras se colocaron en una orientación aproximada, lo que demuestra un conocimiento astronómico en la cultura. Para los sitios cuyos lados tienen valores entre 1 y $6^{\circ}$ de acimut, posiblemente exista alineamiento si se considera la altura del horizonte. Para el resto de lados la diferencia del valor del acimut es bastante grande, por lo que se descarta toda posibilidad de alineamientos al menos relacionados con salidas y puestas del Sol en solsticios yequinoccios.

\section{BIBLIOGRAFÍA}

Aveni, Anthony. (1981). Observadores del cielo en el México antíguo. México: Fondo de Cutura Económica.

Campana, Stefano y Forte, Maurizio. (2006). 2nd International Conference on Remote Sensing in Archaeology: Proceedings of the 2nd International Workshop. Rome: CNR.

Contell, Marina; Gil Piqueras, Teresa; Cortés Alcocer, Paz y Serra Lluch, Juan. (2006). El levantamiento topográfico y arquitectónico. XIX Simposio de Investigaciones Arqueológicas de Guatemala. Guatemala: Universidad Politecnica de Valencia.

Cruz Castillo, Oscar Neill. (2004). Patrón de asentamiento prehispánico en el valle de Jesús de Otoro. VIII Seminario de Antropología Hondureña. Tegucigalpa.

Hawkins, Gerald. (1965). Stonehenge Decoded. Nueva York: Doubleday.

Inestroza, Jesús Evelio. (2007). Jurla en el Valle de Otoro. Historia de Jesús de Otoro y de los pueblos antiguos del valle (1536-2007). Tegucigalpa: Multigráficos Flores. 
Lyons, Thomas R. y Frances, Joan Mathien. (1980). Cultural Resources Remote Sensing. National Washington D.C.: Park Service y Universidad de Nuevo México enAlbuquerque.

McFarlane, Williams y Stockett, Miranda. (2007). Proyecto arqueológico Jesús de Otoro. Informe 2007. Recuperado de: http://scholarspace.jccc.edu/pado/1

Pineda de Carías, María Cristina; Véliz, Vito y Agurcia Fasquelle, Ricardo. (2002). Acerca de las observaciones del Sol realizadas en la Gran Plaza del Parque Arqueológico de Copán Ruinas, Honduras. Yaxkin, XXI, 15-44. Tegucigalpa.

Pineda de Carías, María Cristina; Véliz, Vito y Agurcia Fasquelle, Ricardo. (2002a). El grande y complejo plan de 18 Conejo para la construcción de la Plaza del Sol del Parque Arqueológico de Copán, Honduras. Revista IHAH, Edición Conmemorativa del Instituto Hondureño de Antropología e Historia, 126-134. Tegucigalpa.

Rejas Ayuga, Juan Gregorio. (2008). Desarrollo en nuevas tecnologías para la protección y preservación del patrimonio cultural y hábitats humanos de Honduras. Proyecto: PCI-AECID No. A/019450/08.

Sánchez, Alexis. (2009). Análisis multitemporal de la cobertura de la tierra en la cuenca del Valle de Jesús de Otoro entre los años 2000 - 2006, aplicando técnicas de teledetección. Tesis. UNAH. Tegucigalpa.

Véliz, Vito. (1983). Síntesis histórica de la arqueología en Honduras. Yaxkin, (VI)1 y 2,1-8, Tegucigalpa.

Véliz, Vito. (2010). Arqueología de las zonas de estudio de un proyecto de teledetección en la República de Honduras. FACES-UNAH. Tegucigalpa.

Véliz, Vito; Corrales, Rafael y Castellanos, Yeny. (2009). Proyecto teledetección: informe de avance. FACES-UNAH. Tegucigalpa. 